\title{
Development of Holistic Homogeneous Model of Selective Laser Melting based on Lattice Boltzmann Method: Qualitative Simulation
}

\author{
Dmytro Svyetlichnyy ${ }^{1, \text { a) }}$ and Michal Krzyzanowski ${ }^{1,2, b)}$ \\ ${ }^{1}$ AGH University of Science and Technology, Al. Mickiewicza 30, 30-059 Cracow, Poland \\ ${ }^{2}$ Birmingham City University, Millennium Point, Curzon St., Birmingham B4 7XG, United Kingdom

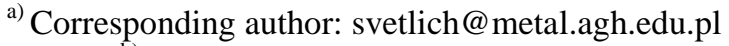 \\ michal.krzyzanowski@bcu.ac.uk
}

\begin{abstract}
Additive manufacturing (AM) technologies are developing fast in recent years. Many of them, such as Selective Laser Sintering/Melting (SLS/SLM) and also Laser Cladding, deal with materials in different states of matter that are changed during processing. The holistic numerical model based on Lattice Boltzmann and cellular automata methods (LBM-CA) is currently being developed for simulation of the laser assisted AM processes where changes of the physical state of matter are essential. The presented qualitative results are mainly related to melting and solidification of the powder bed under the influence of a moving laser beam considering free surface flow, wettability, surface tension and other relevant physical phenomena showing effectiveness of the proposed holistic and homogeneous modelling approach. In this work, we also discuss the potential 3D extension and applications of the model.
\end{abstract}

\section{INTRODUCTION}

SLS/SLM uses a moving laser beam to trace and selectively sinter/melt powdered non-metal and/or metal composite materials into a three-dimensional part of the final product. The product is constructed in the chamber located on a moveable platform that adjusts in height equal to the thickness of the forming layer. Powder for SLS/SLM is added from powder feed supply and deposited on the top of the powder bed over previously proceeded layer of the product and unprocessed powder. Then, a new layer is melted or sintered with a laser beam. The cycles are repeated until the final product is manufactured.

A new holistic numerical model based on Lattice Boltzmann and cellular automata methods (LBM-CA) is currently under development within a frame of an integrated modelling approach applied for studying the complex relationship between different physical mechanisms taking place during laser assisted $\mathrm{AM}{ }^{1}$. The entire process has been analyzed and divided on several stages. The results presented at earlier stages of the development included the entire structure of the model consisting of the different modules connected together demonstrating the homogeneity of the proposed holistic approach ${ }^{1,2}$. The modules, considering several physical phenomena such as powder bed deposition, laser energy absorption, heating of the powder bed by the moving laser beam leading to powder melting or sintering, fluid flow in the melted pool, flow through partly or not melted materials, and solidification were developed to different extent leaving many aspects of this complex integrated numerical approach for further consideration and analysis. 


\section{PROCESS ANALYSIS AND DEVELOPMENT OF HOLISTIC MODEL}

SLS/SLM technological process can be schematically represented with a cyclic process shown in Fig. 1 (the top part ${ }^{1}$. The process can be described as the following sequence: powder deposition - pause - laser beam treatment pause. This sequence can be repeated many times until the product is finished.

The following five basic processes are considered and can be consequently modelled (middle part of Fig.2):

- $\quad$ particles movement,

- $\quad$ laser beam heat exchange and transfer (LBT),

- $\quad$ melting $(\mathrm{M})$,

- free flow of melted material (LF - liquid flow),

- $\quad$ solidification $(\mathrm{S})$.

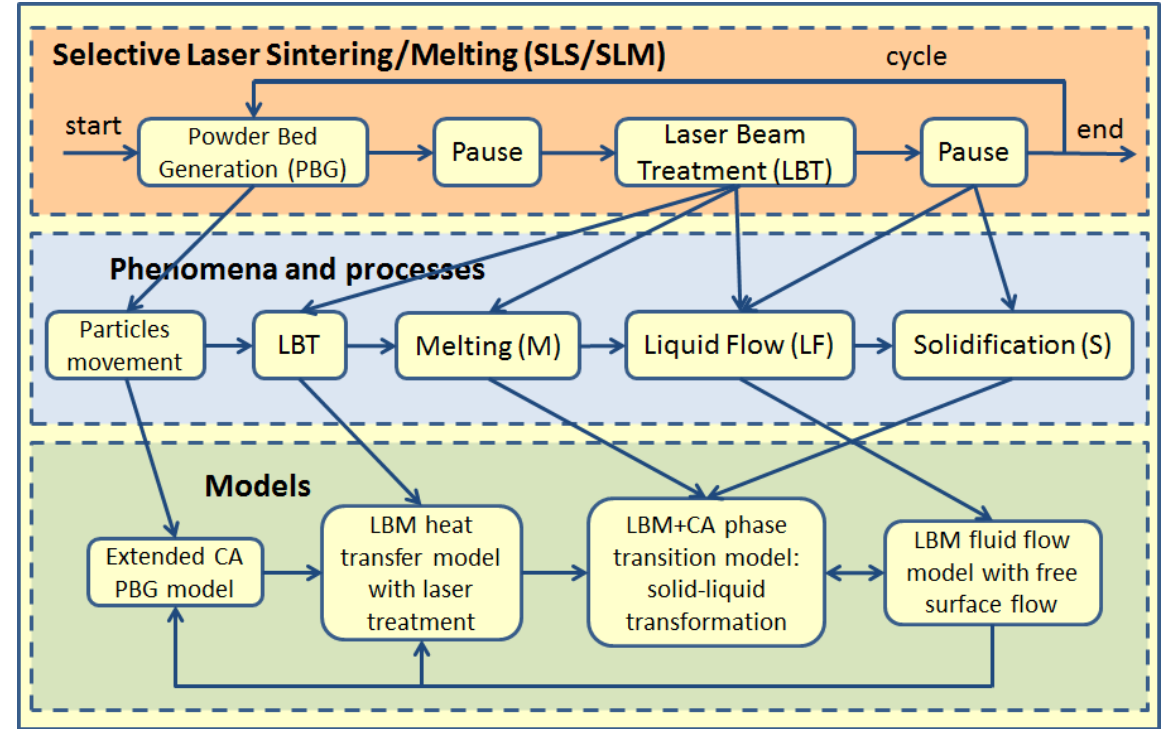

FIGURE 1. Block diagram of SLS/SLM technological process including associated physical processes and models ${ }^{2}$

The model under the current development is a holistic model based entirely on homogeneous methods ${ }^{1}$. The model eliminates complicated interfaces and allows for modelling very complex physical phenomena. Preliminary analysis of the different methods, their benefits and limitations, has shown that one of the appropriate approaches can be based on Lattice Boltzmann and Cellular Automata numerical methods (LBM-CA). A conception of the holistic model and the relevant calculation platform has been discussed earlier presenting a foundation platform and several two-dimensional models ${ }^{1,2}$. Some of the models were connected for modelling of the complex physical phenomena proving feasibility and effectiveness of such holistic modelling approach.

The benefits of the approach are obvious. All models operate in the common domain. A number of processes and phenomena that can be modelled simultaneously are defined by the number of variables associated with a point, site, cell or a node in the same domain. Implementation of a new process or phenomenon requires only addition of new variables, appropriate algorithms and codes.

The defined processes and phenomena associated with SLS/SLM are modelled using the following four submodels considering correspondingly four main tasks (bottom part of Fig. 1), namely:

1. Extended cellular automata model of powder bed generation (PBG).

2. Lattice Boltzmann - heat transfer model of laser treatment.

3. Phase transition model based on LBM and CA.

4. LBM model of fluid flow with free surface.

\section{QUALITATIVE SIMULATION OF SELECTIVE LASER MELTING}

Powder bed generation. The model of powder bed generation was developed on the base of cellular automata ${ }^{3}$. The model has been verified comparing the obtained simulation and experimental results after deposition of Ti-6Al- 
$4 \mathrm{~V}$ powder in a cylinder. The obtained results were in good agreement showing acceptable tolerance (error of packing density about $3.2 \%)^{3}$. Then, the developed code was extended including generation of arbitrary shape particles, more flexible control of particle characteristics and their distribution function ${ }^{3}$.

Heating by laser beam and melting. In SLM process, a laser beam scans on the top of the powder bed as a source of heat influencing radiation, light absorption, scattering, dispersion and diffraction of light in a media. The model of light propagation applied in this work combines elements of LBM and CA. The algorithm similar to particle tracing methods, the methods currently applied for visualization and computer graphics, is used in the modelling approach. It is assumed that the laser radiation transmits light energy in gas as straight beam without scattering. Striking the surface of liquid or solid phase, the energy is divided on the three following parts: reflected, absorbed and transmitted. If the material is opaque, the beam is not transmitted and the radiation is reflected and absorbed. The reflected energy is not considered further in the present version of the model. The light energy penetrating the material is subjected to scattering and absorption. It has to be noted that the interaction between a powder bed and a laser beam is more complicated and the model is going to be extended at later stages to meet requirements of practical applications.

Material flow. Flow of the molten material is considered within a frame of the free surface LBM model assuming gravity, varying surface tension, viscosity and wettability. At present, the model is developed in its $2 \mathrm{D}$ version utilizing sequential calculations in CPU. While 3D model utilizing much faster parallel calculations in GPU is currently under development. In the presented 2D solution, D2Q9 velocity model is used for simulation of thermal and material flow. The 3D model is based on D3Q19 velocity model.

Thermal LBM model with phase transition. The relevant sub-models consider an equation for thermal (diffusion-convection) problem. It takes into account heat flow, heat exchange, heat source and enthalpy of the phase transition.

Different examples of such simulation using the developed sub-models are presented elsewhere ${ }^{2,3}$. The modelling takes into account surface tension reflecting influence of wetting angle, liquid flow on an inclined plane, natural convection, melting and solidification and more complex processes considering heating, melting, various flows and solidification.

Modelling results. In this work, the results of the simulation of the whole SLM cycle are presented for the first time (Fig. 2). The powder bed cross section with two layers of spherical particles is used as an initial state for the simulation. The vertically orientated laser beam moves from the left side to the right side of the domain. The particles have varied transparency. They are assumed almost opaque in the solid state and much more transparent when the state is changed from solid to liquid. The transparency is changed near the transformation temperature. Various values of laser power were applied in the simulations. In Fig. 2, the examples of two laser beam power applications are presented during two consecutive stages of SLM. Solid material is presented by grey color while liquid material by the blue one. The laser beam is shown by the green color. The intensity of heat transfer from the laser beam to material is presented by the variation of pink and red colors. The laser power is twice as higher for the second simulation case presented in Fig 2b, d comparing with the first case (Fig. 2a, c). It can be seen that the amount of melted material is very sensitive to the applied laser power and also the sizes of the melted pool, such as depth and length, depend on the applied laser power.

The obtained results presented in this work can be considered as an outcome of the qualitative rather than qualitative numerical analysis. Nevertheless, they demonstrate possibilities and benefits of the holistic homogeneous model. The simulations do not require complicated interfaces between different sub-models and allow for continuous simulation of the whole processing cycle. The applied modelling space was $160 \times 100$ cells or nodes. A single simulation case lasted about 3h using PC with Intel Core i7-3930K and CPU with sequential code. Several sub-models have been recoded for parallel computations on GPU with CUDA technology using the graphic card GeForce 1060 with 1280 CUDA cores. Application of such parallel calculations allows for 100 times acceleration at least.

\section{Summary}

The holistic homogeneous model based on Lattice Boltzmann method with elements of cellular automata methodology devoted to modelling of SLM process is briefly presented in the paper. The detailed description of the relevant sub-models and their verification are presented elsewhere. This paper presents the simulation results obtained for the first time after combining of all developed sub-models into a single holistic model with the aim to prove feasibility and possible benefits of such holistic approach in modelling of the laser assisted AM process. The 
aim has been successfully achieved showing the qualitative results related to simulation of the whole processing SLM cycle. The model has been developed as 2D version for sequential calculations. 3D version of the model appropriate for parallel calculations is currently being developed. Concurrently, studies leading to introduction of different material properties into the model are carried out that will allow approaching quantitative modelling of a specific selective laser melting process.

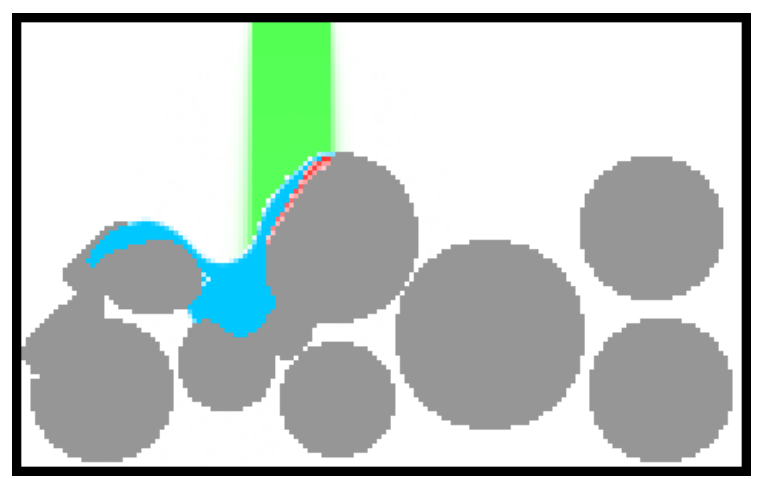

(a)

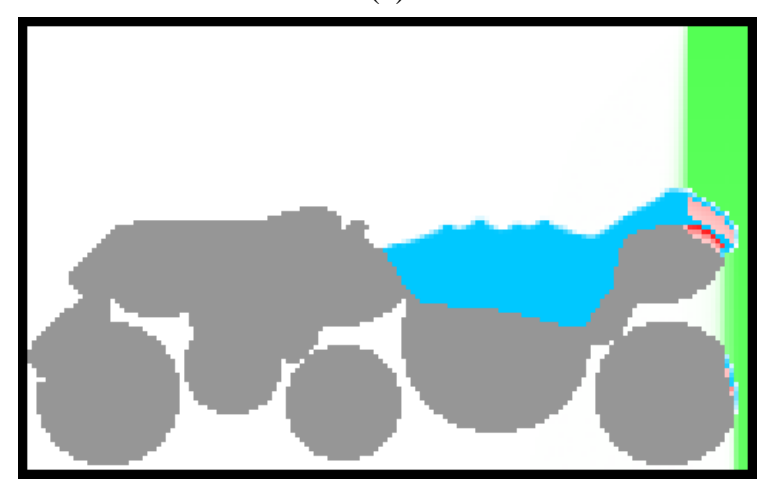

(c)

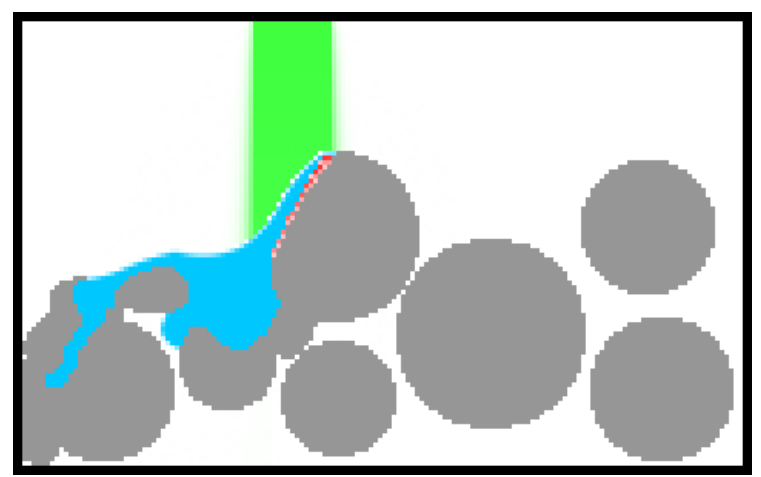

(b)

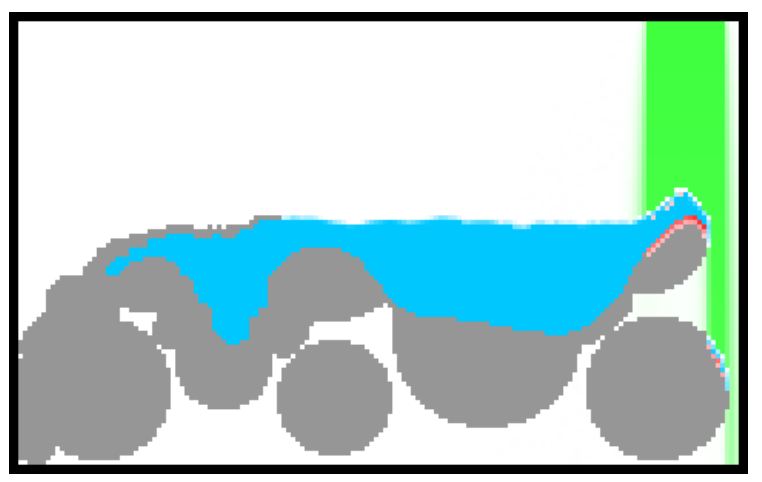

(d)

FIGURE 2. Simulation results of one single cycle of SLM for two different laser power $(a, c)-(b, d)$ at two different stages of the process $(a, b)-(c, d)$

\section{ACKNOWLEDGMENTS}

The support of National Science Centre, Poland, project no. UMO-2018/31/B/ST8/00622 is greatly appreciated.

\section{REFERENCES}

1. D. Svyetlichnyy, M. Krzyzanowski, R. Straka, L. Lach, and W.M. Rainforth, Int. J. Numer. Methods Heat Fluid Flow 28, 31-46 (2018).

2. D. Svyetlichny, A. V. Perig, Ł. Łach, R. Straka, and A. Svyetlichnyy, Int. J. Contin. Eng. Educ. Life-Long Learn. 29, 151-181 (2019).

3. M. Krzyzanowski, D. Svyetlichnyy, G. Stevenson, and W.M. Rainforth, Int. J. Adv. Manuf. Technol. 87, 519530 (2016). 